\title{
Ergodicity test of the eddy-covariance technique
}

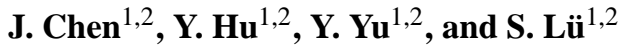 \\ ${ }^{1}$ Key Laboratory of Land Surface Processes and Climate Change in Cold and Arid Regions, Cold and Arid Regions \\ Environment and Engineering Institute, Chinese Academy of Sciences, Lanzhou 730000, China \\ ${ }^{2}$ Pingliang Land Surface Process \& Severe Weather Research Station, Chinese Academy of Science, \\ Pingliang 744015, China
}

Correspondence to: Y. Hu (hyq@ns.lzb.ac.cn)

Received: 27 March 2014 - Published in Atmos. Chem. Phys. Discuss.: 10 July 2014

Revised: 16 April 2015 - Accepted: 7 August 2015 - Published: 4 September 2015

\begin{abstract}
The ergodic hypothesis is a basic hypothesis typically invoked in atmospheric surface layer (ASL) experiments. The ergodic theorem of stationary random processes is introduced to analyse and verify the ergodicity of atmospheric turbulence measured using the eddy-covariance technique with two sets of field observational data. The results show that the ergodicity of atmospheric turbulence in atmospheric boundary layer (ABL) is relative not only to the atmospheric stratification but also to the eddy scale of atmospheric turbulence. The eddies of atmospheric turbulence, of which the scale is smaller than the scale of the ABL (i.e. the spatial scale is less than $1000 \mathrm{~m}$ and temporal scale is shorter than $10 \mathrm{~min}$ ), effectively satisfy the ergodic theorems. Under these restrictions, a finite time average can be used as a substitute for the ensemble average of atmospheric turbulence, whereas eddies that are larger than ABL scale dissatisfy the mean ergodic theorem. Consequently, when a finite time average is used to substitute for the ensemble average, the eddy-covariance technique incurs large errors due to the loss of low-frequency information associated with larger eddies. A multi-station observation is compared with a singlestation observation, and then the scope that satisfies the ergodic theorem is extended from scales smaller than the ABL, approximately $1000 \mathrm{~m}$ to scales greater than about $2000 \mathrm{~m}$. Therefore, substituting the finite time average for the ensemble average of atmospheric turbulence is more faithfully approximate the actual values. Regardless of vertical velocity or temperature, the variance of eddies at different scales follows Monin-Obukhov similarity theory (MOST) better if the ergodic theorem can be satisfied; if not it deviates from MOST. The exploration of ergodicity in atmospheric turbulence is doubtlessly helpful in understanding the issues in at-
\end{abstract}

mospheric turbulent observations and provides a theoretical basis for overcoming related difficulties.

\section{Introduction}

The basic principle of average of the turbulence measurements is based on ensembles averaged over space, time and state. However, it is impossible to make an actual turbulence measurement with enough observational instruments in space for sufficient time to obtain all states of turbulent eddies to achieve the goal of an ensemble average. Therefore, based on the ergodic hypothesis, the time average of one spatial point, taken over a sufficiently long observational time, is used as a substitute for the ensemble average for temporally steady and spatially homogeneous surfaces (Stull, 1988; Wyngaard, 2010; Aubinet et al., 2012). The ergodic hypothesis is a basic assumption in turbulence experiments in the atmospheric boundary layer (ABL) and atmospheric surface layer (ASL). Stationarity, homogeneity, and ergodicity are routinely used to link ensemble statistics (mean and higherorder moments) of field experiments in the ABL. Many authors habitually refer to the ergodicity assumption with descriptions such as "when satisfying ergodic hypothesis..." or "something indicates that ergodic hypothesis is satisfied" (Stull, 1988; Wyngaard, 2010; Aubinet et al., 2012) . The success of Monin-Obukhov similarity theory (MOST) for unstable and near-neutral conditions is just evidence of the validity of the ergodic hypothesis in the ASL. Ergodicity is a necessary condition for the success of MOST, but it does not prove ergodicity (Katul et al., 2004). The success of MOST under the conditions of stationary and homogeneity implies 
that the stationary and homogeneity are also important conditions of ASL ergodicity. Therefore, many ABL experiments focus on seeking ideal homogeneous surfaces. Some test procedures are widely applied to establish stationarity (Foken and Wichura, 1996; Vickers and Mahrt, 1997). Katul and Hsieh (1999) qualitatively analysed the ergodicity problem in atmospheric turbulence and believed that it is common for the neutral and unstable ASL to satisfy ergodicity, while it is difficult to reach ergodicity in the stable ASL. Eichinger et al. (2001) indicate that the lidar technique opens up new possibilities for atmospheric measurements and analyses by providing spatial and temporal atmospheric information with simultaneous high resolution. The stationarity and ergodicity can be tested for such ensembles of experiments. Recent advances in lidar measurements offer a promising first step for direct evaluation of such hypotheses for ASL flows (Higgins et al., 2013). Higgins et al. (2013) applied lidar of water vapour concentration to investigate the ergodic hypothesis of atmospheric turbulence for the first time. It is clear all the same that there is a need to reevaluate the technologies of turbulence measurement, to test the ergodicity of atmospheric turbulence quantitatively by means of observation experiments.

The ergodic hypothesis was first proposed by Boltzmann (Boltzmann, 1871; Uffink, 2004) in his study of the ensemble theory of statistical dynamics. He argued that a trajectory traverses all points on the energy hypersurface after a certain amount of time. At the beginning of 20th century, the Ehrenfest couple (Ehrenfest and Ehrenfest-Afanassjewa, 1912; Uffink, 2004) proposed a quasi-ergodic hypothesis and changed the term "traverses all points" in the aforesaid ergodic hypothesis to "passes arbitrarily close to every point". The basic points of ergodic hypothesis or quasi-ergodic hypothesis recognize that the macroscopic property of a system in the equilibrium state is an average of microcosmic quantity in a sufficiently long time. Nevertheless, the ergodic hypothesis or quasi-ergodic hypothesis was never proven theoretically. The proof of the ergodic hypothesis in physics aroused the interest of mathematicians. Famous mathematician, Neumann et al. (1932) first theoretically proved the ergodic theorem in topological space (Birkhoff, 1931; Krengel, 1985). Afterward, a banausic ergodic theorem of stationary random processes was proven to provide a necessary and sufficient condition for the ergodicity of stationary random processes. Mattingly (2003) reviewed the research progress on ergodicity for stochastically forced Navier-Stokes equation, and that Galanti and Tsinober (2004) and Lennaert et al. (2006) solved the Navier-Stokes equation by numerical simulation to prove that turbulence that is temporally steady and spatially homogeneous is ergodic. However, Galanti and Tsinober (2004) also indicated that such partially turbulent flows acting as mixed layer, wake flow, jet flow, flow around the boundary layer may be non-ergodic.

Obviously, the advances of research on ergodicity in mathematics and physics have led the way for atmospheric sci- ences. We try first to introduce the ergodic theorem of stationary random processes to the atmospheric turbulence in this paper. The ergodicity of different scale eddies of atmospheric turbulence is directly analysed and verified quantitatively on the basis of field observation data obtained using eddy-covariance technique in the ASL.

\section{Theories and methods}

\subsection{Ergodic theorems of stationary random processes}

Stationary random processes are processes which will not vary with time; that is, for observed quantity $A$, its function of space $x_{i}$ and time $t_{i}$ satisfies the following condition:

$A\left(x_{1}, x_{2}, \ldots, x_{n} ; t_{1}, t_{2}, \ldots, t_{n}\right)=A\left(x_{1}, x_{2}, \ldots, x_{n}\right.$;

$\left.t_{1}+\tau, t_{2}+\tau, \ldots, t_{n}+\tau\right)$,

where $\tau$ is a time period, defined as the relaxation time.

The mean $\mu_{A}$ of a random variable $A$ and its autocorrelation function $R_{A}(\tau)$ are, respectively, defined as follows:

$\mu_{A}=\lim _{T \rightarrow+\infty} \frac{1}{T} \int_{0}^{T} A(t) \mathrm{d} t$,

$R_{A}(\tau)=\lim _{T \rightarrow+\infty} \frac{1}{T} \int_{0}^{T} A(t) A(t+\tau) \mathrm{d} t$.

The autocorrelation function $R_{A}(\tau)$ is a temporal secondorder moment. In the case of $\tau=0$, the autocorrelation function $R_{A}(\tau)$ is the variance of random variable. A necessary and sufficient condition for the stationary random processes to satisfy the mean ergodicity is the mean ergodic function $\operatorname{Ero}(A)$ to 0 (Papoulis and Pillai, 1991), as shown below:

$\operatorname{Ero}(A)=\lim _{T \rightarrow \infty} \frac{1}{T} \int_{0}^{2 T}\left(1-\frac{\tau}{2 T}\right)\left[R_{A}(\tau)-\mu_{A}^{2}\right] \mathrm{d} \tau=0$.

The mean ergodic function $\operatorname{Ero}(A)$ is a time integral of the difference between the autocorrelation function $R_{A}(\tau)$ of variable $A$ and its mean square, $\mu_{A}^{2}$. If the mean ergodic function $\operatorname{Ero}(A)$ converges to 0 , then the stationary random processes will be ergodic. In other words, if the autocorrelation function $R_{A}(\tau)$ of variable $A$ converges to its mean square, $\mu_{A}^{2}$, the stationary random processes are mean ergodic. Equation (4) is namely mean ergodic theorem, also known as ergodic theorem of the weakly stationary processes in mathematics. For discrete variables, Eq. (4) can be rewritten as follows:

$\operatorname{Ero}(A)=\lim _{n \rightarrow \infty} \sum_{i=0}^{n}\left(1-\frac{\tau_{i}}{n}\right)\left[R_{A}\left(\tau_{i}\right)-\mu_{A}^{2}\right]=0$. 
Equation (4) is mean ergodic theorem of the discrete variable. Hence, Eq. (4) or (5) can be used as a criterion to judge the mean ergodicity.

For the stationary random processes, the necessary and sufficient condition satisfying the autocorrelation ergodicity is the autocorrelation ergodic function $\operatorname{Er}(A)$ to 0 :

$\operatorname{Er}(A)=\lim _{T \rightarrow \infty} \frac{1}{T} \int_{0}^{2 T}\left(1-\frac{\tau^{\prime}}{2 T}\right)\left[B\left(\tau^{\prime}\right)-\left|R_{A}(\tau)\right|^{2}\right] \mathrm{d} \tau^{\prime}=0 ;$

$B\left(\tau^{\prime}\right)=E\left\{A\left(t+\tau+\tau^{\prime}\right) A\left(t+\tau^{\prime}\right)[A(t+\tau) A(t)]\right\}$,

where $\tau$, is a differential variable for entire relaxation times, and $B(\tau \prime)$ is temporal fourth-order moment of variable $A$. The autocorrelation ergodic function $\operatorname{Er}(A)$ is a time integral of the difference between the temporal fourth-order moment $B(\tau \prime)$ of variable $A$ and its autocorrelation function square, $\left|R_{A}(\tau)\right|^{2}$. If the autocorrelation ergodic function $\operatorname{Er}(A)$ converges to 0 , then the stationary random processes will be of autocorrelation ergodicity, and thus the autocorrelation ergodicity means that the fourth-order moment of variable of stationary random processes will converge to square of its autocorrelation function $R_{A}(\tau)$. Equation (6a) is namely autocorrelation ergodic theorem, also known as ergodic theorem of the strongly stationary processes in mathematics. The autocorrelation ergodic function of corresponding discrete variable can be determined as follows:

$\operatorname{Er}(A)=\lim _{n \rightarrow \infty} \sum_{i=0}^{n}\left(1-\frac{\tau_{i}^{\prime}}{n}\right)\left[B\left(\tau_{i}^{\prime}\right)-\left|R_{A}\left(\tau_{j}\right)\right|^{2}\right]=0$,

$B\left(\tau_{i}^{\prime}\right)=E\left\{\sum_{j=0}^{n} A\left(t+\tau_{j}+\tau_{i}^{\prime}\right) A\left(t+\tau_{i}^{\prime}\right)\left[A\left(t+\tau_{j}\right) A(t)\right]\right\}$.

Equation (7a) is autocorrelation ergodic theorem of the discrete variable. Hence, Eq. (6a) or (7a) can also be used as a criterion to judge the autocorrelation ergodicity.

The stationary random processes conform to the criterion, Eq. (4) or (5); then they satisfy the mean ergodic theorem or are intituled as the mean ergodicity. The stationary random processes conform to the criterion, Eq. (6a) or (7a); then they satisfy the autocorrelation ergodic theorem or are intituled as the autocorrelation ergodicity. If the stationary random processes are only of mean ergodicity, they are strictly ergodic or narrowly ergodic. If the stationary random processes are of both the mean ergodicity and autocorrelation ergodicity, they are namely wide ergodic stationary random processes. It is thus clear that the ergodic random processes are stationary, but the stationary processes may not be ergodic.

In the random process theory, calculating the mean or high-order moment function requires a large number of repeated observations to acquire a sample function $A_{k}(t)$. If the stationary random processes satisfy the ergodic condition, then time average of a sample on the whole time span can be used to substitute for the ensemble average. Equations (4), (5), (6a) and (7a) can be used as the criteria to judge whether or not the mean and autocorrelation ergodicity are satisfied. The ergodic random processes must be the stationary random processes to be defined as Eq. (1) and thus are stationary in relaxation time $\tau$. If the condition such as Eq. (4) or (5) of the mean ergodicity is satisfied, then a time average in finite relaxation time $\tau$ can be used to substitute for infinite time average to calculate the mean Eq. (2) of random variable; similarly, the finite time average can be used for substitution to calculate the covariance or variance of random variable, Eq. (3), if the condition such as Eq. (6a) or (7a) of autocorrelation ergodicity is satisfied. In a similar manner, the basic principle of average of the atmospheric turbulence is the ensemble average of space, time and state, and it is necessary to carry out mass observations for a long period of time in the whole space. Not only is this a costly observation, but it is also barely feasible. If the turbulence satisfies the ergodic condition, then a time average in relaxation time $\tau$ by multi-station observation, even single-station observation, can substitute for the ensemble average. In fact, precondition to estimate turbulent characteristic quantities and fluxes in the ABL by the eddy-covariance technique is that the turbulence satisfies the ergodic condition. Therefore, conditions such as Eqs. (4), (5), (6a) and (7a) will also be the criteria for testing the ergodicity and authenticity of results observed by the eddy-covariance technique.

\subsection{Band-pass filtering}

The scope of spatial and temporal scale of the atmospheric turbulence, which is from the dissipation range, inertial subrange to the energy range, and further the turbulent large eddy, is extremely broad (Stull, 1988). In such wide spatial and temporal scope, the turbulent eddies include the isotropic 3-D eddy structure of high-frequency turbulence and orderly coherent structure of low-frequency turbulence ( $\mathrm{Li}$ et al., 2002). These eddies of different scale are also different from each other in terms of their spatial structure and physical properties, and even their transport characteristics are not the same. It is thus reasonable that eddies with different characteristics are separated, processed and studied using different methods (Zuo et al., 2012). A major goal of our study is to understand what type of eddy in the scale can satisfy the ergodic condition. Another goal is that the time averaging of signals measured by a single station determines accurately turbulent characteristic quantities. In order to study the ergodicity of different scale eddies, Fourier transform is used as a band-pass filtering to distinguish different scale eddies. That is to say, we aim to set the Fourier transform coefficient of the part of frequencies, which it does not need, as 0 , and then we acquire the signals after filtering by means of Fourier inverse transformation. The specific formulae are shown be- 
low:

$$
\begin{aligned}
& F_{A}(n)=\frac{1}{N} \sum_{k=0}^{N-1} A(k) \cos \left(\frac{2 \pi n k}{N}\right) \\
& -\frac{i}{N} \sum_{k=0}^{N-1} A(k) \sin \left(\frac{2 \pi n k}{N}\right), \\
& A(k)=\sum_{n=a}^{N-1} F_{A}(n) \cos \left(\frac{2 \pi n k}{N}\right) \\
& +i^{2} \sum_{n=a}^{N-1} F_{A}(n) \sin \left(\frac{2 \pi n k}{N}\right) .
\end{aligned}
$$

In Eqs. (8) and (9), $F_{A}(n)$ and $A(k)$ are, respectively, the Fourier transformation and Fourier inverse transformation including $N$ data points from $k=0$ to $k=N-1$, and $n$ is the cycle index of the observation time range. The highpass filtering can cut off the low-frequency signals of turbulence to obtain the high-frequency signals. An aliasing of half high-frequency turbulence after the Fourier transformation is unavoidable. At this time, the correction for highfrequency response will compensate for that loss. In order to acquire purely signals of different scale eddies in filtering processes, we take results of the band-pass filtering from $n=j$ to $n=N-j$ as required signals. This is referred to as $j$ time filtering in this paper. Finally, the ergodicity of different scale eddies is analysed using Eqs. (4)-(7).

\subsection{MOS of turbulent variance}

The characteristics of the relations of Monin-Obukhov similarity (MOS) for the variance of different scale eddies are analysed and compared to test feasibility of the MOS relations for ergodic and non-ergodic turbulence. In order to provide an experimental basis for utilizing MOST and developing the turbulence theory of $\mathrm{ABL}$ under the condition of the complex underlying surfaces, the problems of eddycovariance technique of the turbulence observation in ASL are further explored on the basis of studying the ergodicity and MOS relations of the variance of different scale eddies.

The MOS relations of turbulent variance can be regarded as an effective instrumentality to verify whether or not the turbulent flow field is steady and homogeneous (Foken et al., 2004). Under ideal conditions, the local MOS relations of the variance of wind velocity, temperature and other factors can be expressed as follows:

$\sigma_{i} / u_{*}=\varphi_{i}(z / L),(i=u, v, w)$,

$\sigma_{s} /\left|s_{*}\right|=\varphi_{s}(z / L),(s=\theta, q)$,

where $\sigma$ is turbulent variance; corner mark $i$ is wind velocity $u, v$ or $w$; $s$ stands for scalar, such as potential temperature $\theta$ and humidity $q, u_{*}$ is friction velocity and defined as $u_{*}=\left({\overline{u^{\prime} w^{\prime}}}^{2}+{\overline{v^{\prime} w^{\prime}}}^{2}\right)^{1 / 4} ; s_{*}$ is turbulent characteristic quan- tity related to scalar defined as $s_{*}=-\overline{w^{\prime} s^{\prime}} / u_{*}$; and MoninObukhov length $L$ is defined as the following (Hill, 1989):

$L=u_{*}^{2} \theta /\left[\kappa g\left(\theta_{*} /+0.61 \theta q_{*} / \rho_{\mathrm{d}}\right)\right]$,

where $\rho_{\mathrm{d}}$ is dry air density.

A large number of research results show that, in the case of unstable stratification, $\varphi_{i}(z / L)$ and $\varphi_{s}(z / L)$ can be expressed in the following forms (Panofsky et al., 1977; Padro 1993; Katul et al., 1999):

$\varphi_{i}(z / L)=c_{1}\left(1-c_{2} z / L\right)^{1 / 3}$

$\varphi_{s}(z / L)=\alpha_{s}\left(1-\beta_{s} z / L\right)^{-1 / 3}$,

where $c_{1}, c_{2}, \alpha$ and $\beta$ are coefficients to be determined by the field observation. In the case of stable stratification, $\varphi_{s}(z / L)$ approximates a constant and $\varphi_{i}(z / L)$ is still the $1 / 3$ function of $z / L$. The turbulent characteristics of eddies in different temporal and spatial scale are analysed and compared with the mean and autocorrelation ergodic theorems, to test feasibility of MOS relations under the condition of the ergodic and non-ergodic turbulence.

\section{The sources and processing of data}

In this study two turbulence data sets are used for completely different purposes. The first turbulence data set is the data measured by the eddy-covariance technique under the homogeneous surface in Nagqu Station of Plateau Climate and Environment (NSPCE), Chinese Academy of Sciences (CAS). The data set in NSPCE/CAS includes the data that are measured by 3-D sonic anemometer and thermometer (CSAT3) with $10 \mathrm{~Hz}$ as well as infrared gas analyser (Li7500) in ASL from 23 July to 13 September 2011. In addition, the second turbulence data set of CASES-99 (Poulos et al., 2002; Chang and Huynh, 2002) is used to verify the ergodicity of turbulence observed by multiple stations. CASES-99 has seven observation sites, equivalent to seven observation stations. The data in the central tower of CASES-99 include those measured by sonic anemometer and thermometer (CSAT3) with $20 \mathrm{~Hz}$ and the infrared gas analyser (Li7500) at $10 \mathrm{~m}$ on a tower with $55 \mathrm{~m}$ height in ASL. The other six subsites of CASES-99 surrounding the central tower, sn1, sn2 and sn3 are located $100 \mathrm{~m}$ are away from the central tower, the subsite sn 4 is $280 \mathrm{~m}$ away, and subsites sn5 and sn6 are located $300 \mathrm{~m}$ away. The data of subsites include those measured by 3-D sonic anemometer (ATI) and Li7500 at $10 \mathrm{~m}$ height on the towers. The analysed results with two data sets are compared to each other to test universality of the research results.

The geographic coordinate of NSPCE/CAS is $31.37^{\circ} \mathrm{N}$, $91.90^{\circ} \mathrm{E}$, and its altitude is $4509 \mathrm{~m}$ a.s.l. The observation station is built on a flat and wide area except for a hill of about $200 \mathrm{~m}, 2 \mathrm{~km}$ to the north, and floor area is $8000 \mathrm{~m}^{2}$. The ground surface is mainly composed of sandy soil mixed 
with sparse fine stones, and a plateau meadow with vegetation of $10-20 \mathrm{~cm}$. The roughness length and displacement height of underlying surface of NSPCE meadow are, respectively, 0.009 and $0.03 \mathrm{~m}$. CASES-99 is located in a prairie of Kansas, United States. The geographic coordinates of CASES-99 central tower are $37.65^{\circ} \mathrm{N}, 96.74^{\circ} \mathrm{W}$. The observation field is flat and grass growth of about $20-50 \mathrm{~cm}$ during the observation period, while the roughness length and displacement height of the CASES-99 underlying surface are 0.012 and $0.06 \mathrm{~m}$, respectively (Martano, 2000).

These data are used to study the ergodicity of turbulent eddies in ABL. Firstly the inaccurate data caused by spike are deleted before data analyses. Subsequently, the data are divided into continuous sections of $5 \mathrm{~h}$, and the signals of $1 \mathrm{~h}$ are obtained applying filtering of Eqs. (8) and (9) for each $5 \mathrm{~h}$ of data. In order to delete further the abnormal inaccurate data, the data are divided once again into 12 continuous fragments of $5 \mathrm{~min}$ in $1 \mathrm{~h}$. The variances of velocity and temperature are calculated and compared to each other for the fragments. The data with a deviation of less than $\pm 15 \%$, including an instrumental error about $\pm 5 \%$, are selected to use. Moreover, temperature of the ultrasonic pulse signals is converted to the absolute temperature (Schotanus et al., 1983; Kaimal and Gaynor, 1991). Then all data without spike for 25 days undergo a coordinate rotation using the plane fitting method to improve the levelness of instrument installation (Wilczak, 2001). The trend correction (McMillen, 1988; Moore, 1986) is used to exclude the influence of lowfrequency trend effect caused by the diurnal variations and weather processes. The Webb correction (Webb et al., 1980) is a component of surface energy balance in physical nature but not the component of turbulent eddy. However, this study analyses the ergodicity of turbulent eddies. According to our preliminary analysis of the ergodicity of turbulent eddies, such a correction may cause an unreasonable deviation from the prediction with Eq. (14). We thus do not perform the Webb correction in our research on ergodicity.

\section{Result analyses}

Applying the two data sets from NSPCE/CAS and CASES99, the ergodicity of different temporal scale eddies is tested. Here as an example, we select representative data measured at a level of $3.08 \mathrm{~m}$ in NSPCE/CAS during three time frames, namely 03:00-04:00, 07:00-08:00 and 13:00-14:00 China standard time (CST) on 25 August under clear-sky conditions to test and demonstrate the ergodicity of different temporal scale eddies. These three time frames represent three situations, i.e. the nocturnal stable boundary layer, early neutral boundary layer and midday convective boundary layer.

Equations (8) and (9) are used to perform band-pass filtering from $n=j$ to $n=N-j$ to acquire the signals of eddies corresponding temporal scale including $2,3,5,10,30$ and $60 \mathrm{~min}$. The turbulence characteristics and ergodicity of ed- dies in the different temporal scale including 2, 3, 5, 10,30 and $60 \mathrm{~min}$ are studied using the data processed above for three time frames.

\subsection{Monin-Obukhov eddy local stability and Monin-Obukhov stratification stability}

The Monin-Obukhov stratification stability parameter $z / L$ describes a whole characteristic of the mechanical and buoyancy effect on the ASL turbulence. However this study will decompose the turbulence into different scale eddies. Considering that the property of different scale eddies of the atmospheric turbulence varies with the atmospheric stability parameter $z / L$, a Monin-Obukhov eddy local stability that is limited in the certain scale range of eddies is defined as $z / L_{\mathrm{c}}$, so as to analyse relations between the stratification stability and ergodicity of the different scale eddies for the wind velocity, temperature and other factors. It is worth noting that the Monin-Obukhov eddy local stability, $z / L_{\mathrm{c}}$, is different from the Monin-Obukhov stratification stability, $z / L$.

As a typical example, the eddy local stabilities, $z / L_{\mathrm{c}}$, of the different temporal scales for the three time frames from the nighttime to the daytime are shown in Table 1 . The results show that the eddy local stability $z / L_{\mathrm{c}}$ below $2 \mathrm{~min}$ at temporal scale at time 03:00-04:00 (CST) during the nighttime time frame is 0.59; thus it is stable stratification. But as the eddy temporal scale gradually increases from 3,5 and 10 to $60 \mathrm{~min}$, the eddy local stability, $z / L_{\mathrm{c}}$, gradually decreases to 0.31 and 0.28 . Even starting from $10 \mathrm{~min}$ in the temporal scale, the eddy local stability decreases from -0.01 to -0.07 . It seems that the eddy local stability gradually varies from stable to unstable as the eddy temporal scale increases. At 07:00-08:00 (CST) during the morning time frame, the eddy local stability $z / L_{\mathrm{c}}$ from 2 to $60 \mathrm{~min}$ at the temporal scale eventually decreases from $0.52,0.38,0.16$ and 0.15 to -0.43 in $30 \mathrm{~min}$ and a minimum of -1.29 in $60 \mathrm{~min}$. This means that eddies at the temporal scales of $30 \mathrm{~min}$ and $60 \mathrm{~min}$ have high local instability. However, at 14:00-15:00 (CST) during the midday time frame, eddies at the temporal scales from 2 to $60 \mathrm{~min}$ are all unstable. Now $-z / L_{\mathrm{c}}$ is defined as eddy local instability. As the eddy scale increases, the eddy local instability in the scales from 2 to $3 \mathrm{~min}$ also increases. In addition, its value reaches a maximum of 0.44 as the eddy scale is at $5 \mathrm{~min}$. But as the eddy scale increases continuously, the eddy local instability is reduced.

The Monin-Obukhov eddy local stability is not entirely the same as the Monin-Obukhov stratification stability of ABL in the physical significance. The Monin-Obukhov stratification stability of ABL indicates the overall effect of atmospheric stratification in the ABL on the stability including all eddies in integral boundary layer. The Monin-Obukhov stratification stability $z / L$ is stable 0.02 at 03:00-04:00 (CST) for no filtering data to include whole turbulent signals, but unstable -0.004 and -0.54 at 07:00-08:00 and 13:00-14:00 (CST), respectively. However the eddy local stability is only 
Table 1. Local stability parameter $(z-d) / L_{\mathrm{c}}$ of the eddies in different temporal scales on 25 August.

\begin{tabular}{lrrr}
\hline \multicolumn{1}{c}{ Time } & $03: 00-04: 00$ & $07: 00-08: 00$ & $14: 00-15: 00$ \\
\hline Eddy scale & & & \\
\hline$\leq 2 \mathrm{~min}$ & 0.59 & 0.52 & -0.38 \\
$\leq 3 \mathrm{~min}$ & 0.31 & 0.38 & -0.44 \\
$\leq 5 \mathrm{~min}$ & 0.28 & 0.16 & -0.40 \\
$\leq 10 \mathrm{~min}$ & -0.01 & 0.15 & -0.34 \\
$\leq 30 \mathrm{~min}$ & -0.04 & -0.43 & -0.27 \\
$\leq 60 \mathrm{~min}$ & -0.07 & -1.29 & -0.30 \\
\hline
\end{tabular}

a local effect of atmospheric stratification on the stability of eddies at a certain scale. As the eddy scale increases, the eddy local stability $z / L_{\mathrm{c}}$ will vary accordingly. The aforesaid results indicate that the local stability of small-scale eddies is stable in the nocturnal stable boundary layer, but it is possibly unstable for the large-scale eddies. As a result, there is a sink effect on the small-scale eddies in the nocturnal stable boundary layer, but there is a positive buoyancy effect on the large-scale eddies. However, in the diurnal unstable boundary layer, the eddy local instability of 3 min scale reaches a maximum, and then the instability gradually decreases as the eddy scale increases. Therefore, eddies of 3 min scale hold maximum buoyancy, but the eddy buoyancy decreases as the eddy scale increases continuously. Nevertheless, the small-scale eddies are more stable than the large-scale eddies in the nocturnal stable boundary layer; the large-scale eddies are more stable than the small-scale eddies in the diurnal convective boundary layer with unstable stratification. The above facts signify that it is common that there exist mainly small-scale eddies in the nocturnal boundary layer with stable stratification. And it is also common that there exist mainly large-scale eddies in the diurnal convective boundary layer with unstable stratification. Therefore, it can well understand that small-scale eddies are dominant in the nocturnal stable boundary layer, while large-scale eddies are dominant in the diurnal convective boundary layer.

\subsection{Verification of mean ergodic theorem of eddies in different temporal scale}

In order to verify the mean ergodic theorem, we calculate the mean and autocorrelation functions using Eqs. (2) and (3), then calculate the variation of mean ergodic function $\operatorname{Ero}(A)$ using Eq. (5) of eddies in the different temporal scale with relaxation time $\tau$ to be cut off with $\tau_{i=n}$. The mean ergodic functions, $\operatorname{Ero}(A)$, of vertical velocity, temperature and specific humidity of the different scale eddies are calculated using data at level of 3.08 $\mathrm{m}$ at 03:00-04:00, 07:00-08:00 and 13:00-14:00 (CST) for three time frames in NSPCE/CAS, as shown in Figs. 1-3, respectively. Since the ergodic function varies within a large range, the ergodic functions are nor- malized according to the characteristic quantity of relevant variables $\left(A_{*}=u_{*},\left|\theta_{*}\right|,\left|q_{*}\right|\right)$. That is to say, functions in all following figures are the dimensionless ergodic functions, $\operatorname{Ero}(A) / A_{*}$.

Comprehensive analyses of the characteristics of mean ergodicity of atmospheric turbulence as well as the relevant causes are discussed in the following sections.

\subsubsection{Verifying mean ergodic theorem of different scale eddies}

According to the mean ergodic theorem, Eq. (4), the mean ergodic function $\operatorname{Ero}(A) / A_{*}$ will converge to 0 if the time approaches infinity. This is only a theoretical result of the stationary random processes. A practical mean ergodic function is calculated under the condition of that relaxation time $\tau_{i=n}$ is cut off. If the mean ergodic function $\operatorname{Ero}(A) / A_{*}$ converges approximately to 0 in relaxation time $\tau_{i=n}$, it will be considered that random variable $A$ approximately satisfies the mean ergodic theorem. If the mean ergodic function deviates more from 0 , the mean ergodicity will be of poor quality. Consequently, we can judge approximately whether or not the mean ergodic theorem of different scale eddies holds. Figures 1-3 clearly show that, regardless of the vertical velocity, temperature or humidity, the $\operatorname{Ero}(A) / A_{*}$ of eddies below $10 \mathrm{~min}$ in the temporal scale will swing around 0 within a small range; thus we can conclude that the mean ergodic function $\operatorname{Ero}(A) / A_{*}$ of eddies below $10 \mathrm{~min}$ at the temporal scale converges to 0 to satisfy effectively the condition of mean ergodic theorem. For eddies of 30 and $60 \mathrm{~min}$, which are larger scale, the mean ergodic function $\operatorname{Ero}(A) / A_{*}$ will deviate further from 0 . In particular, the mean ergodic function $\operatorname{Ero}(A) / A_{*}$ of eddies of 30 and $60 \mathrm{~min}$ for the temperature and humidity does not converge, and even diverges. The results above show that the mean ergodic function of eddies of 30 and 60 min cannot converge to 0 or cannot satisfy the condition of mean ergodic theorem.

\subsubsection{Comparison of the convergence of mean ergodic functions of vertical velocity, temperature and humidity}

As seen from Figs. 1-3, dimensionless mean ergodic function of the vertical velocity is compared with respective function of the temperature and humidity. It is 3-4 orders of magnitude less than those in the nocturnal stable boundary layer; 1-2 orders of magnitude less than those in the early neutral boundary layer; and about 2 orders of magnitude less than those in the midday convective boundary layer. For example, at 15:00-16:00 (CST) during nighttime time frame, the dimensionless mean ergodic function of vertical velocity is $10^{-5}$ in magnitude, while respective magnitudes of function value of the temperature and humidity are $10^{-1}$ and $10^{-2}$; at 07:00-08:00 (CAT) during morning time frame, magnitude of mean ergodic function of the vertical velocity is $10^{-4}$, 


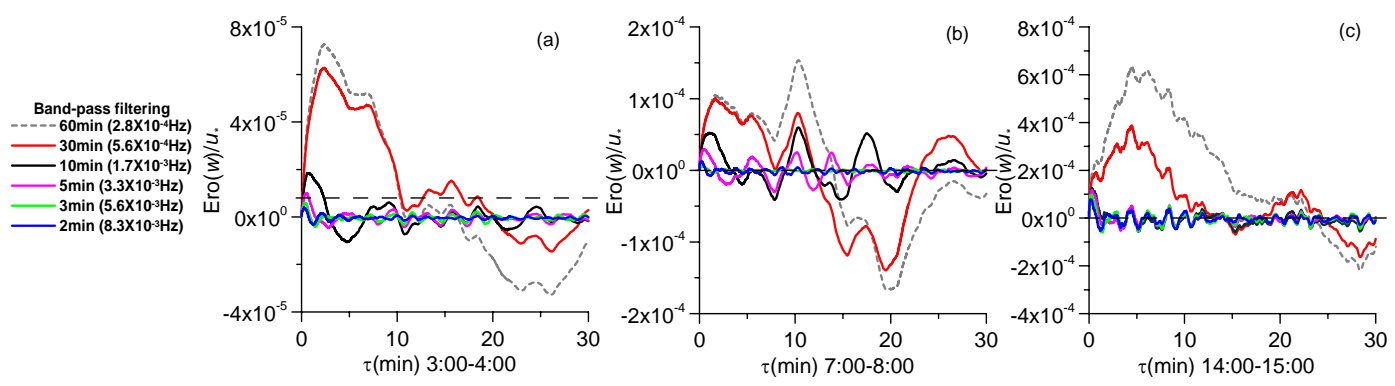

Figure 1. Variation of mean ergodic function $\operatorname{Ero}(w)$ of vertical velocity measured at the height $3.08 \mathrm{~m}$ in NSPCE with relaxation time for the different scale eddies after band-pass filtering. (a), (b) and (c) are the respective results of the three time frames. If their mean ergodic function is more approximate to 0 , then eddies in the corresponding temporal scale will more closely satisfy the ergodic conditions.
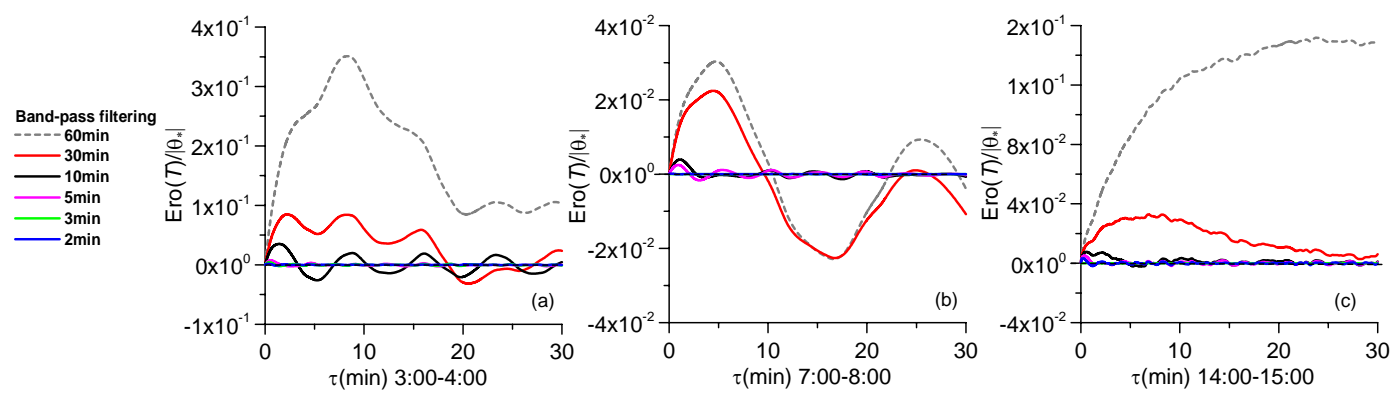

Figure 2. Variation of mean ergodic function $\operatorname{Ero}(T)$ of the different scale eddies of temperature with relaxation time (other conditions are as some as Fig. 2, and the same applies to the following figures).

while the respective magnitudes of function value of the temperature and humidity are $10^{-2}$ and $10^{-3}$; at 13:00-14:00 (CST) during midday time frame, magnitude of mean ergodic function of the vertical velocity is $10^{-4}$, while the magnitudes of function value of the temperature and humidity are both $10^{-2}$. These results show that the dimensionless mean ergodic function of vertical velocity converges to 0 much more easily than respective function value of the temperature and humidity, and that the vertical velocity satisfies the condition of mean ergodic theorem to overmatch more than the temperature and humidity.

\subsubsection{Temporal scale and spatial scale of turbulent eddies}

For wind velocity of $1-2 \mathrm{~ms}^{-1}$, eddy spatial scale at the temporal scale of $2 \mathrm{~min}$ is in the range of $120-240 \mathrm{~m}$, and eddy spatial scale in the temporal scale of $10 \mathrm{~min}$ is in the range of 600-1200 m. The eddy spatial scale at the temporal scale of $2 \mathrm{~min}$ is equivalent to ASL height, and the eddy spatial scale at the temporal scale of $10 \mathrm{~min}$ is equivalent to $\mathrm{ABL}$ height. The eddy spatial scale within the temporal scales of $30-60 \mathrm{~min}$ is around $1800-3600 \mathrm{~m}$, and this spatial scale clearly exceeds ABL height to belong to the scope of the atmospheric local circulation. According to the stationary random processes defined in Eq. (1) and mean ergodic theorem, the stationary random processes must be smooth in relaxation time $\tau$. The eddies below temporal scale of $10 \mathrm{~min}$, i.e. below ABL height, can effectively satisfy the condition of mean ergodic theorem and must be the stationary random processes of mean ergodicity. However, eddies in the temporal scales of 30 and 60 min exceed ABL height and do not satisfy the condition of mean ergodic theorem.

\subsubsection{Turbulence ergodicity of all eddies in possible scales in ABL}

To facilitate comparison, Fig. 4 shows the variation of mean ergodic function $\operatorname{Ero}(A)$ of the vertical velocity (a), temperature (b) and specific humidity (c) before filtering with relaxation time $\tau$ at 14:00-15:00 (CST) during midday time frame in the convective boundary layer. It is obvious that Fig. 4 is unfiltered mean ergodic function of eddies in all possible scales in ABL. Figure 4 compares with Figs. 1c, 2c and $3 \mathrm{c}$, which are the mean ergodic function $\operatorname{Ero}(A) / A_{*}$ of vertical velocity, temperature and humidity after filtering at 14:00-15:00 (CST) during the midday time frame. The result shows that the mean ergodic functions before filtering are greater than those after filtering. As shown in Figs. 1c, 2c and $3 \mathrm{c}$, the magnitude for the vertical velocity is $10^{-4}$ and the magnitudes for the temperature and specific humidity are both $10^{-2}$. According to Fig. 4 , the magnitude of vertical velocity $\operatorname{Ero}(A) / A_{*}$ is $10^{-3}$ and the magnitudes of temperature and specific humidity are both $10^{\circ}$; therefore, $1-2$ orders of 

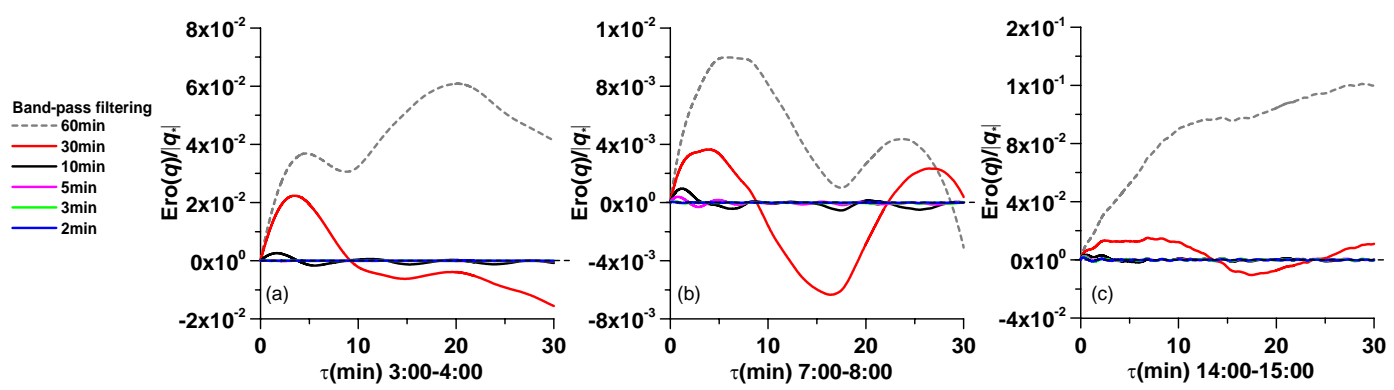

Figure 3. Variation of mean ergodic function $\operatorname{Ero}(q)$ of the different scale eddies of humidity with relaxation time.
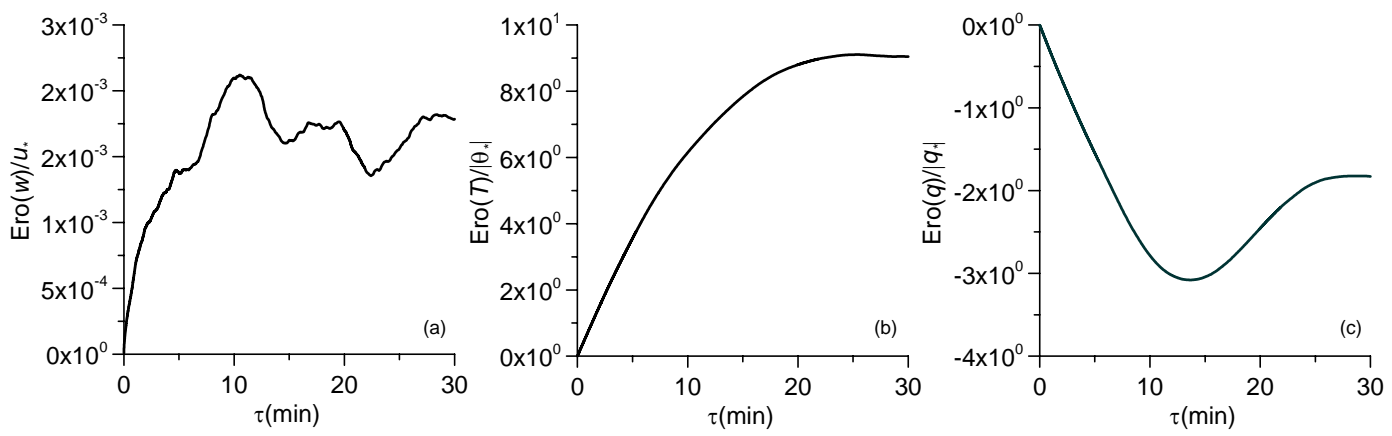

Figure 4. Variation of mean ergodic function $\operatorname{Ero}(w)$ of the vertical velocity (a), temperature (b) and specific humidity (c) before filtering at 14:00-15:00 (CST) during midday in NSPCE with relaxation time $\tau$.

magnitude are almost decreased after filtering. Moreover, all magnitudes trend upward deviating from 0 for vertical velocity and temperature, but they trend downward deviating from 0 for specific humidity. It is thus clear that, at 14:0015:00 (CST) during the midday time frame, equivalent to the 12:00-13:00 local time, the unfiltered mean ergodic function of eddies in all possible scales in the convective boundary layer cannot converge to 0 before filtering, i.e. cannot satisfy the condition of mean ergodic theorem. This may be that eddies in all possible scales before filtering include the local circulation in the convective boundary layer. So we argue that, under general circumstances, the eddies only below $10 \mathrm{~min}$ at the temporal scale or within $600-1200 \mathrm{~m}$ at the spatial scale in ABL must be the stationary random processes of mean ergodicity.

\subsubsection{Relation between the ergodicity and local stability of different scale eddies}

Table 1 lists the corresponding relation of eddy local stabilities $z / L_{\mathrm{c}}$ of eddies of different scales with the different time frames. It shows that the eddy local stabilities $z / L_{\mathrm{c}}$ of different scale eddies are different, due to the fact that the temperature stratification in ABL has a different effect on the stability of different scale eddies. Even entirely contrary results can occur. At the same time, the stratification that causes the large-scale eddy to ascend with buoyancy may cause the small-scale eddy to descend. However, the results in Figs. 1-
3 show that the ergodicity is mainly related to the eddy scale, and its relation to the atmospheric temperature stratification seems secondary.

\subsection{Verification of autocorrelation ergodic theorem for different scale eddies}

In this section, Eqs. (7a) and (7b) are used to verify the autocorrelation ergodic theorem. This is in accordance with Sect. 4.2 that the turbulent eddies below $10 \mathrm{~min}$ at temporal scale satisfy the mean ergodic condition in the various time frames; i.e. the turbulent eddies below $10 \mathrm{~min}$ at temporal scale are at least strictly stationary random processes or narrow stationary random processes - whether in the nocturnal stable boundary layer, in the early neutral boundary layer, or midday convective boundary layer. Then we analyse further the different scale eddies that satisfy the mean ergodic condition and whether or not they also satisfy the autocorrelation ergodic condition, so as to verify whether or not atmospheric turbulence is a narrow or wide stationary random process. The autocorrelation ergodic function of turbulence variable $A$ under the condition of truncated relaxation time $\tau_{i=n}$ is calculated according to Eq. (7a) to determine the variation of autocorrelation ergodic function $\operatorname{Er}(A)$ with relaxation time $\tau$. As with the mean ergodic function $\operatorname{Ero}(A)$, if the autocorrelation ergodic function $\operatorname{Er}(A)$ of eddies of 2, 3, 5, 10, 30 and $60 \mathrm{~min}$ at the temporal scale within the relaxation time $\tau_{i=n}$ approximates 0 , then $A$ shall be deemed approx- 

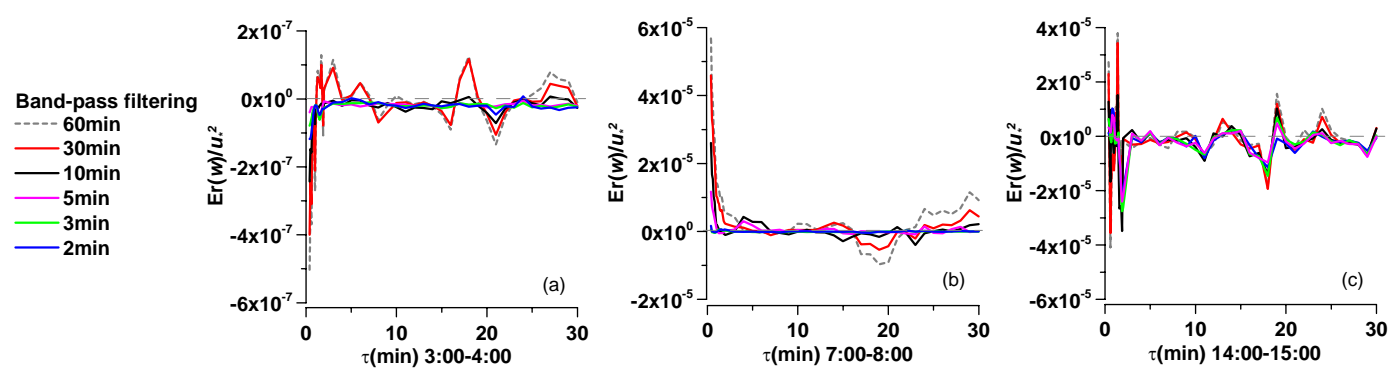

Figure 5. Variation of the autocorrelation ergodic function of vertical velocity with relaxation time for different scale eddies.

imately ergodic; the more the autocorrelation ergodic function deviates from 0 , the worse the autocorrelation ergodicity becomes. Therefore, this method can be used to judge approximatively whether the different scale eddies satisfy the condition of autocorrelation ergodic theorem.

As an example of the vertical velocity, Fig. 5 shows the variation of normalized autocorrelation ergodic function $\operatorname{Er}(w) / u_{*}$ of the turbulent eddies of 2, 3, 5, 10,30 and $60 \mathrm{~min}$ at the temporal scale with relaxation time $\tau$ at 03:00-04:00, 07:00-08:00 and 13:00-14:00 (CST) during the time frames, respectively. Some basic conclusions are drawn from Fig. 5 as follows:

1. After comparing Fig. 5a-c with Fig. 1a-c, i.e. comparing the dimensionless mean ergodic function $\operatorname{Ero}(w) / u_{*}$ of vertical velocity with the dimensionless autocorrelation ergodic function $\operatorname{Er}(w) / u_{*}$, two basic characteristics are very clear. First, the magnitudes of the dimensionless autocorrelation ergodic function $\operatorname{Er}(w) / u_{*}$, regardless of whether in the nocturnal stable boundary layer, early neutral boundary layer or midday convective boundary layer, are all greatly reduced. In Fig. 1ac, the magnitudes of $\operatorname{Ero}(w) / u_{*}$ are, respectively, $10^{-5}$, $10^{-4}$ and $10^{-4}$, and the magnitudes of $\operatorname{Er}(w) / u_{*}$ are, respectively, $10^{-7}, 10^{-5}$ and $10^{-5}$ as shown in Fig. 5a-c. The magnitudes of $\operatorname{Er}(w) / u_{*}$ are reduced by $1-2$ compared with those of $\operatorname{Ero}(w) / u_{*}$. Second, all autocorrelation ergodic functions $\operatorname{Er}(w) / u_{*}$ of the eddies of $30 \mathrm{~min}$ and $60 \mathrm{~min}$ at temporal scale, regardless of whether they are in the stable boundary layer, natural boundary layer or convective boundary layer, are all reduced and approximate to $\operatorname{Er}(w) / u_{*}$ of the eddies below $10 \mathrm{~min}$ at temporal scale.

2. The above two basic characteristics imply that the autocorrelation ergodic function $\operatorname{Er}(w) / u_{*}$ of the stable boundary layer, neutral boundary layer or convective boundary layer converges to 0 faster than the mean ergodic function $\operatorname{Ero}(w) / u_{*}$; the autocorrelation ergodic function of eddies of 30 and $60 \mathrm{~min}$ at temporal scale also converges to 0 and satisfies the condition of autocorrelation ergodic theorem, except for the fact that the autocorrelation ergodic function $\operatorname{Er}(w) / u_{*}$ of the eddies below $10 \mathrm{~min}$ at temporal scale can converge to 0 and satisfy the condition of autocorrelation ergodic theorem.

3. According to the autocorrelation ergodic function Eq. (7a), the eddies of 30, 60 and below $10 \mathrm{~min}$ at the temporal scale, regardless of whether they are in the stable boundary layer, neutral boundary layer or convective boundary layer, all eddies satisfy the condition of autocorrelation ergodic theorem. Therefore, in general ABL turbulence is the stationary random process of autocorrelation ergodicity.

4. The above results show that the eddies below $10 \mathrm{~min}$ at temporal scale in the nocturnal stable boundary layer, early neutral boundary layer and midday convective boundary layer satisfy not only the condition of mean ergodic theorem but also the condition of autocorrelation ergodic theorem. Therefore, eddies below $10 \mathrm{~min}$ at the temporal scale are wide ergodic stationary random processes. Although the eddies of 30 and $60 \mathrm{~min}$ at temporal scale in the stable boundary layer, neutral boundary layer and convective boundary layer satisfy the condition of autocorrelation ergodic theorem, they dissatisfy the condition of mean ergodic theorem. Therefore, eddies of 30 and $60 \mathrm{~min}$ at the temporal scale are neither narrow ergodic stationary random processes nor wide ergodic stationary random processes.

\subsection{Ergodic theorem verification of different scale eddies for the multi-station observations}

The basic principle of turbulence average is an ensemble average of the space, time and state. Sections 4.2 and 4.3 verify the mean ergodic theorem and autocorrelation ergodic theorem of atmospheric turbulence using field observational data, so that the finite time average of a single station can be used to substitute for the ensemble average for the ergodic turbulence. This section examines the ergodicity of different scale eddies using the observational data of a centre tower and six subsites of CASES-99, in all seven sites, equivalent to seven stations. When the data are selected, the following is considered: if the eddies are not evenly distributed at the seven sites, then the observation results at the seven sites 

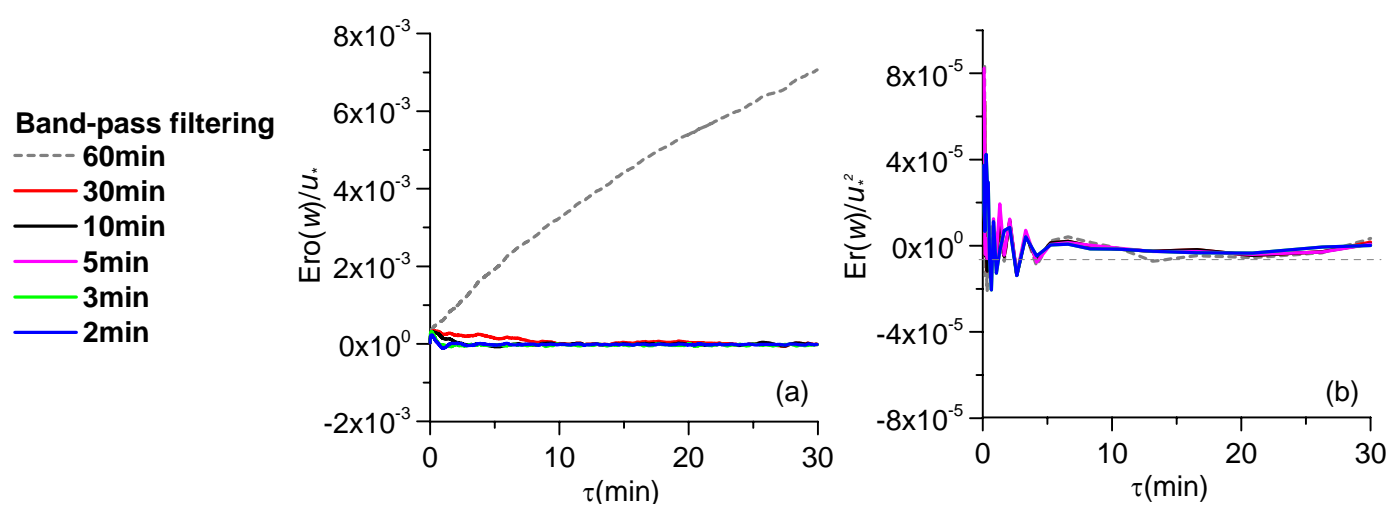

Figure 6. Variation of mean ergodic function (a) and autocorrelation ergodic function (b) of the vertical velocity with relaxation time for the different scale eddies in the seven stations of CASES-99.

may have originated from many eddies at a large scale. For this reason, the high-frequency variance spectrum in excess of $0.1 \mathrm{~Hz}$ is compared firstly. Based on the observational error, if the scatter of all high-frequency variances does not exceed the average by $\pm 10 \%$, then it is assumed that the turbulence is evenly distributed at the seven observation sites. Then, 17 data sets are chosen from among the observed turbulence data from 5 to 30 October, and these data sets represent typical strong turbulence at noon on the sunny day. As an example, the same method as described in Sects. 4.2 and 4.3 is used to, respectively, calculate variation of the mean ergodic function and autocorrelation ergodic function with relaxation time $\tau$ for the vertical velocity at 10:00-11:00 on 7 October. The time series composed of the above data sets is performed band-pass filtering in 2, 3, 5, 10,30 and $60 \mathrm{~min}$. The variations of mean ergodic function $\operatorname{Ero}(w) / u_{*}$ and autocorrelation ergodic function $\operatorname{Er}(w) / u_{*}$ with relaxation time $\tau$ are analysed for the vertical velocity to test the ergodicity of different scale eddies for observations of the multiple stations. Figure 6a shows variation of mean ergodic function $\operatorname{Ero}(w) / u_{*}$ with the relaxation time $\tau$ for the vertical velocity, and Fig. $6 \mathrm{~b}$ shows variation of autocorrelation ergodic function $\operatorname{Er}(w) / u_{*}$ with the relaxation time $\tau$.

The results show ergodic characteristics of different scale eddies measured with multi-station observations as follows: Fig. 6a shows that the mean ergodic function of eddies below $30 \mathrm{~min}$ at temporal scale converges to 0 very well, except for the fact that the mean ergodic function of eddies of $60 \mathrm{~min}$ at temporal scale clearly deviates upward from 0 . Figure $6 \mathrm{~b}$ shows that autocorrelation ergodic function of all different scale eddies, including $60 \mathrm{~min}$ at temporal scale, gradually converges to 0 . Therefore, eddies below $30 \mathrm{~min}$ at temporal scale measured with the multi-station observations satisfy the conditions of both the mean and autocorrelation ergodic theorem, while eddies of $60 \mathrm{~min}$ at temporal scale only satisfy the condition of autocorrelation ergodic theorem but dissatisfy the condition of mean ergodic theorem. These facts demonstrate that eddies below $30 \mathrm{~min}$ at temporal scale are the wide ergodic stationary random processes for time series of the above data sets composed by the seven stations. This signifies that, comparing data composed of the multi-station observations with data from a single station, the eddy temporal scale of wide ergodic stationary random processes is extended from below 10 to $30 \mathrm{~min}$. As analysed above, if the eddies below $10 \mathrm{~min}$ at temporal scale are deemed the turbulent eddies in the ABL with height of about $1000 \mathrm{~m}$, then the eddies of $30 \mathrm{~min}$ at the temporal scale, equivalent to the space scale greater than $2000 \mathrm{~m}$, are deemed including eddy components of the local circulation in ABL. Therefore the multi-station observations can completely capture the local circulated eddies, which space scale is greater than $2000 \mathrm{~m}$.

\subsection{Average time problem of turbulent quantity averaging}

The atmospheric observations are impossible to repeat experiments exactly, must use the ergodic hypothesis and replace ensemble averages with time averages. The problem of how to determine the averaging time arises.

The analyses on the ergodicity of different scale eddies in the above two sections demonstrate that the eddies below $10 \mathrm{~min}$ at temporal scale as relaxation time $\tau=30 \mathrm{~min}$ in the stable boundary layer, neutral boundary layer and convective boundary layer satisfy not only the mean ergodic theorem but also the autocorrelation ergodic theorem. That is to say, they are namely wide ergodic stationary random processes. Therefore, a finite time average of $30 \mathrm{~min}$ within relaxation time $\tau$ can be used for substitution of the ensemble average to calculate mean random variable, Eq. (2). However, the eddies of 30 and $60 \mathrm{~min}$ at temporal scale in the stable boundary layer and neutral boundary layer are only autocorrelation ergodic random processes, neither narrow nor wide sense random processes. Therefore, when the finite time average of $30 \mathrm{~min}$ is used for substitution of the ensemble average to calculate mean random variable Eq. (2), it may capture the eddies below $10 \mathrm{~min}$ at temporal scale in stationary random processes, but it cannot completely capture the eddies in ex- 

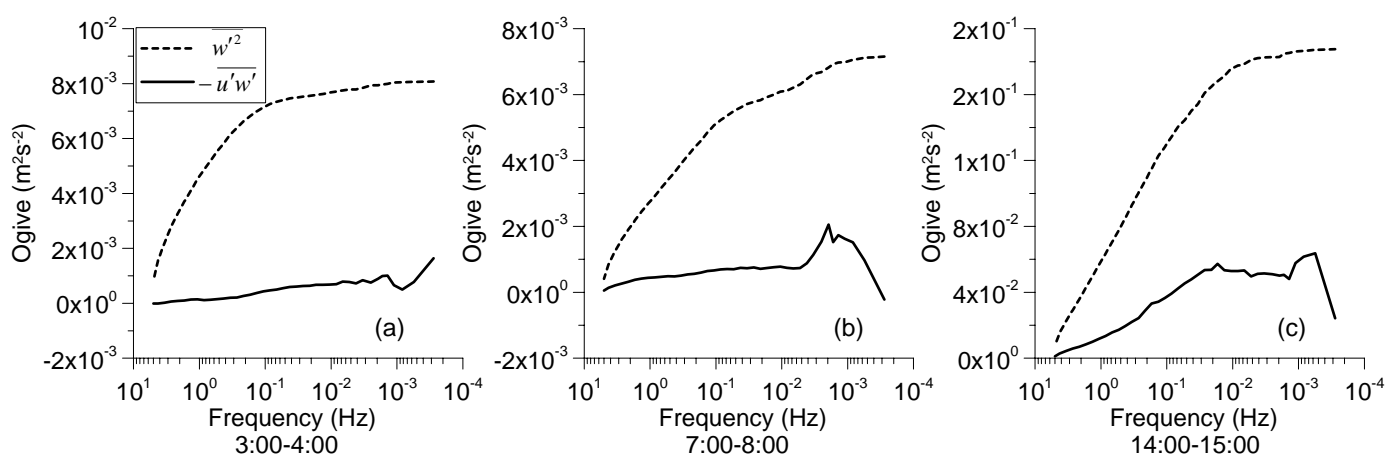

Figure 7. Variation of ogive functions of $\overline{w^{\prime 2}}$ and $-\overline{u^{\prime} w^{\prime}}$ with frequency at height $3.08 \mathrm{~m}$ for the three time frames in NSPCE.

cess of 30 min at temporal scale. The above results signify that the turbulence average is not only restricted by the mean ergodic theorem but also closely related to the scale of turbulent eddies. In the atmospheric observations performed using the eddy-covariance technique, the substitution of ensemble average with finite time average of $30 \mathrm{~min}$ inevitably results in a high level of error, due to loss of low-frequency component information associated with the large-scale eddies. However, although eddies of $30 \mathrm{~min}$ and $60 \mathrm{~min}$ at temporal scale in the convective boundary layer are not wide ergodic stationary random processes, they are autocorrelation ergodic random processes. This may imply that the mean of atmospheric turbulence in the convective boundary layer, which is calculated to substitute the finite time average for the ensemble average, is often superior to the results of the stable boundary layer and neutral boundary layer. In addition, the results in the previous sections also show that the mean ergodic function of vertical velocity may more easily converge to 0 than functions corresponding to the temperature and humidity; i.e. the vertical velocity may more easily satisfy the condition of mean ergodic theorem than the temperature and humidity. Therefore, in the observation performed using the eddy-covariance technique, the result of vertical velocity is often superior to those of the temperature and humidity. In the previous section, the results also point out that multistation observations can completely capture eddies of the local circumfluence in the ABL. Therefore, the multi-station observation is more likely to satisfy the ergodic assumption, and its results are much closer to the true values. In order to determine the averaging time, Oncley (1996) defined an ogive function of cumulative integral

$O g_{x, y}\left(f_{0}\right)=\int_{\infty}^{f_{0}} \operatorname{Co}_{x, y}(f) \mathrm{d} f$,

where $x$ and $y$ are any two variables, and their covariance is $\bar{x} \bar{y}$, and $\mathrm{Co}_{x y}(f)$ is the cospectrum of $x y$. If the ogive function converges to a constant value at a frequency $f=f_{0}$, this frequency could be converted to an averaging time. Ogive function of $\overline{u^{\prime} w^{\prime}}$ is often used to examine the minimal av- eraging time. As a comparison, here the variation of ogive functions of $\overline{w^{\prime 2}}$ and $\overline{u^{\prime} w^{\prime}}$ with frequency at the height $3.08 \mathrm{~m}$ in NSPCE/CAS for the three time frames is shown in Fig. 7. Figure 7 shows convergence frequency of ogive function for $\overline{w^{\prime 2}}$ in the nighttime stable boundary layer, morning-tide neutral boundary layer and midday convection boundary layer is, respectively, about at $0.01,0.0001$ and $0.001 \mathrm{~Hz}$. It is equivalent to the averaging times about 2,160 and $16 \mathrm{~min}$. For $\overline{u^{\prime} w^{\prime}}$, it converges about at $0.001 \mathrm{~Hz}$ only in the midday convection boundary layer, equivalent to the averaging time of about $16 \mathrm{~min}$; it seems there is no convergence in the nighttime stable and morning-tide neutral boundary layer. It is implied that determination of the averaging time encounters a bit of difficulty with the ogive function in the stable and neutral boundary layer. Figure 7 shows also that when the frequency is lower than $0.0001 \mathrm{~Hz}$, ogive functions $\overline{u^{\prime} w^{\prime}}$ ascend in the stable boundary layer, but they descend in the morningtide neutral boundary layer and midday convection boundary layer. We must especially note that the ogive function is a cumulative integral. Therefore, as the ogive function changes direction from ascending to descending, it implies a possibility that there exists a superimposing of the negative and positive momentum fluxes caused by a cross local circulation effect in nighttime and midday. This cross local circulation in ABL may cause the low-frequency effect on the ogive function so that the local circulation in ABL may be an important reason why ogive fails to judge the averaging time. In this work, the choice of averaging time with the ergodic theory seems superior to that with the ogive function.

\subsection{MOS of turbulent eddies in different scales and its relation to ergodicity}

Turbulent variance is a most basic characteristic quantity of the turbulence. Turbulence velocity variance, which represents turbulence intensity, and the variance of scalars, such as temperature and humidity, effectively describes the structural characteristics of turbulence. In order to test MOS relation of the different scale eddies with ergodicity, the vertical velocity and temperature data of NSPCE/CAS from 23 July 
Table 2. Parameters of the fitting curve of MOS relation for vertical velocity variance.

\begin{tabular}{rrrrrrr}
\hline & \multicolumn{2}{c}{$10 \min$} & \multicolumn{2}{c}{$30 \mathrm{~min}$} & \multicolumn{2}{c}{$60 \mathrm{~min}$} \\
\hline & $z / L<0$ & $z / L>0$ & $z / L<0$ & $z / L>0$ & $z / L<0$ & $z / L>0$ \\
\hline$c_{1}$ & 1.08 & 1.17 & 1.06 & 1.12 & 0.98 & 1.06 \\
$c_{2}$ & 4.11 & 3.67 & 3.64 & 3.27 & 4.62 & 2.62 \\
$R$ & 0.97 & 0.76 & 0.94 & 0.56 & 0.83 & 0.30 \\
$S$ & 0.19 & 0.25 & 0.17 & 0.27 & 0.25 & 0.31 \\
\hline
\end{tabular}

to 13 September are used to determine the MOS relationship of variances of vertical velocity and temperature for the different scale eddies, and to analyse its relation to the ergodicity.

The MOS relation of vertical velocity variance is as follows:

$\varphi_{i}(z / L)=c_{1}\left(1-c_{2} z / L\right)^{1 / 3}, z / L<0$,

$\varphi_{i}(z / L)=c_{1}\left(1+c_{2} z / L\right)^{1 / 3}, z / L>0$.

Figures 8 and 9, respectively, show the MOS relation curves of different scale eddies for the vertical velocity and temperature variances in NSPCE/CAS. The panels $a, b$ and $c$ of Figs. 8 and 9 are, respectively, the similarity curve of eddies of 10, 30 and $60 \mathrm{~min}$ at temporal scale. Table 2 shows the relevant parameters of fitting curve of MOS relation for the vertical velocity variance. The correlation coefficient and residual of fitting curve are, respectively, expressed with $R$ and $S$.

Figure 8 and Table 2 show that the parameters of fitting curve are greatly different, even if the fitting curve modality of MOS relation of the vertical velocity variance is the same for the eddies in different temporal scales. The correlation coefficients of MOS fitting curve of the vertical velocity variance under the unstable stratification are large, but the correlation coefficients under the stable stratification are small. Under unstable stratification, the correlation coefficient of eddies of $10 \mathrm{~min}$ in the temporal scale reaches 0.97 , while the residual is only 0.16 ; under the stable stratification, the correlation coefficient reduces to 0.76 , and the residual increases to 0.25 . With the increase of eddy temporal scale from 10 (Fig. 8a) to $30 \mathrm{~min}$ (Fig. 8b) and $60 \mathrm{~min}$ (Fig. 8c), the correlation coefficients of MOS relation of the vertical velocity variance gradually reduce, and the residuals increase. The correlation coefficient in $60 \mathrm{~min}$ reaches a minimum; it is 0.83 under the unstable stratification, and only 0.30 under the stable stratification.

The temperature variance is shown in Fig. 9. MOS function to fit from eddies of $10 \mathrm{~min}$ at the temporal scale under the unstable stratification is as follows:

$\varphi_{\theta}\left(z / L_{\mathrm{c}}\right)=4.9\left(1-79.7 z / L_{\mathrm{c}}\right)^{-1 / 3}$.

As shown in Fig. 9a, the correlation coefficient of fitting curve is 0.91 and the residual is 0.38 . With an increase of the eddy temporal scale, discreteness of MOS relation of the temperature variance is increased quickly to incur that the appropriate curve cannot be fitted.

The above results show that the discreteness of fitting curve of MOS relation for the turbulence variance is increased with the increase of eddy temporal scale, whether it is the vertical velocity or temperature. The points of data during the stationary processes basically gather near the fitting curve of variance similarity relation, while all data points during the non-stationary processes deviate significantly from the fitting curve. However, the similarity of vertical velocity variance is superior to that of the temperature variance. These results are consistent with the conclusions of ergodicity test for the different scale eddies described in Sects. 2-4.4. The ergodicity of the small-scale eddies is superior to that of the larger-scale eddies, and eddies of $10 \mathrm{~min}$ at the temporal scale have the best variance similarity relations. These results also signify that when eddies in the stationary random processes satisfy the ergodic condition, both the vertical velocity variance and temperature variance of eddies in the different temporal scales comply with MOST very well but, as for eddies with poor ergodicity during non-stationary random processes, the variances deviate from MOS relations.

\section{Discussions}

1. Galanti and Tsinober (2004) proved that the turbulence, which is temporally steady and spatially homogeneous, is ergodic, but "partially turbulent flows" such as the mixed layer, wake flow, jet flow, flow around and boundary layer flow may be non-ergodic turbulence. However, it has been proven through atmospheric observational data that the turbulence ergodicity is related to the scale of turbulent eddies. Since the large-scale eddies in ABL may be strongly influenced by the boundary disturbance, they thus belong to "partial turbulence"; however, since the small-scale eddies in atmospheric turbulence may not be influenced by boundary disturbance, they may be temporally steady and spatially homogeneous turbulence so that the mean ergodic theorem and autocorrelation ergodic theorem are applicable for turbulence eddies at the small scale in ABL, but the ergodic theorems are not applicable for the large-scale eddies (i.e. the small-scale eddies in the ABL are ergodic and the large-scale eddies exceeding the ABL scale are nonergodic).

2. The eddy-covariance technique for turbulence measurement is based on the ergodic assumption. A lack of ergodicity related to the presence of large-scale eddy transport can lead to a considerable error of the flux measurement. This has already been pointed out by Mauder et al. (2007) or Foken et al. (2011). Therefore, we realize from the above results that the large-scale eddies that exceed ABL height may include components 

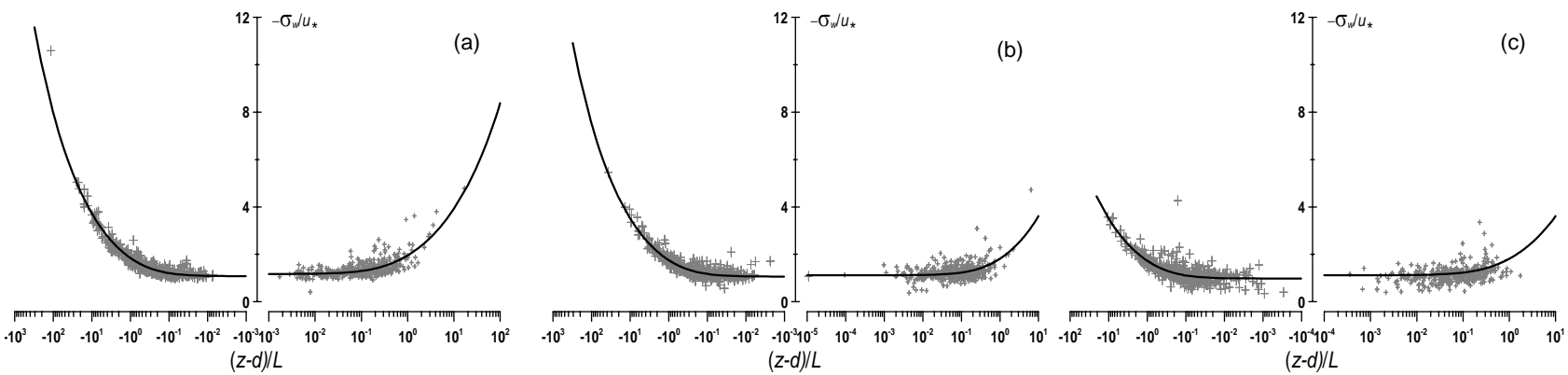

Figure 8. MOS relation of vertical velocity variances of the different scale eddies in NSPCE; (a), (b) and (c), respectively, represent the similarity of eddies of 10,30 and $60 \mathrm{~min}$ at the temporal scale.
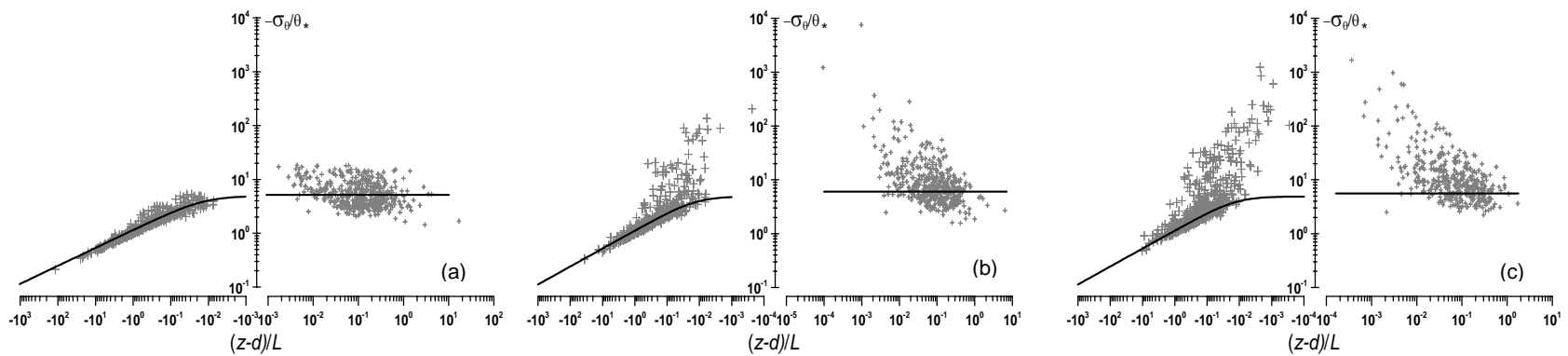

Figure 9. MOS relations of temperature variance of in different scale eddies of NSPCE; (a), (b) and (c), respectively, represent the similarity of the eddies of 10, 30 and $60 \mathrm{~min}$ at the temporal scale.

of non-ergodic random processes. The eddy-covariance technique cannot capture the signals of large-scale eddies exceeding ABL scale to result in the large error in the measurements of atmospheric turbulent variance and covariance. MOST is developed under the condition of steady time and a homogeneous surface. MOST conditions, steady time and homogeneous underlying surface are in line with the ergodic conditions. Therefore, the turbulence variances, even the turbulent fluxes of eddies at different temporal scales, may comply with MOST very well, if the ergodic conditions of stationary random processes are more effectively satisfied.

3. According to Kaimal and Wyngaard (1990), the atmospheric turbulence theory and observation method were feasible and led to success under ideal conditions including a short period, steady state and homogeneous underlying surface, and through observation in the 1950s-1970s, but these conditions are rare in reality. In the land surface processes and ecosystem, the turbulent flux observations in ASL turn into a scientific issue. Commonly, there are interested researchers in the fields of atmospheric sciences, ecology, geography sciences, etc. These observations must be implemented under conditions such as complex terrain, heterogeneous surface, long period and unsteady state. It is necessary that more neoteric observational tools and theories be applied with new perspectives in future research.
4. The ergodic theorem of stationary random processes has successfully been introduced from mathematics into atmospheric sciences. It undoubtedly provides a profitable tool for overcoming the challenges encountered in the modern measurements of atmospheric turbulent flow. At least it offers a promising first step to diagnosticate directly the ergodic hypotheses for ASL flows as a criterion. The necessary and sufficient condition of ergodic theorem can be used to judge the applicable scope of eddy-covariance technique and MOST, as well as seek potential disable reasons for using them in the ABL.

5. In the future, we shall continue our study of the ergodic problems for the atmospheric turbulence measurements under the conditions of complex terrain, heterogeneous surface and unsteady, long observational period, as well as seek effective schemes. The above results indicate that the atmospheric turbulent eddies below the scale of ABL can be captured by the eddy-covariance technique and comply with MOST very well. Perhaps MOST can be a first-order approximation to deal with the turbulence of eddies below ABL scale in order to satisfy the ergodic theorems, to compensate for the effects of eddies dissatisfying the ergodic theorem, which may be caused by the advection, local circulation, lowfrequency effect, etc. under the complex terrain, heterogeneous surface. For example, we developed a turbu- 
lent theory of non-equilibrium thermodynamics $(\mathrm{Hu}$, et al., 2007; Hu and Chen, 2009) to find the coupling effects of vertical velocity, which is caused by the advection, local circulation, and low frequency, on the vertical fluxes. The coupling effects of vertical velocity may be a scheme to compensate for the effects of eddies dissatisfying the ergodic theorems (Hu, 2003; Chen, et al., 2007, 2013).

6. It is clear that such studies are preliminary, and many problems require further research. The attestation of more field experiments is necessary.

\section{Conclusions}

From the above results, we can draw preliminary conclusions:

1. The turbulence in ABL is an eddy structure. When the temporal scale of turbulent eddies in ABL is about $2 \mathrm{~min}$, the corresponding spatial scale is about 120 $240 \mathrm{~m}$, equivalent to ASL height; when the temporal scale of turbulent eddies in ABL is about $10 \mathrm{~min}$, the corresponding spatial scale is about 600-1200 m, equivalent to the ABL height. For the eddies of larger temporal and spatial scale, such as eddies of 30-60 min at the temporal scale, the corresponding spatial scale is about 1800-3600 m to exceed the ABL height.

2. The above results show that the ergodicity of atmospheric turbulence in ABL is relative not only to the atmospheric stratification but also to the eddy scale of atmospheric turbulence. For the atmospheric turbulent eddies below the ABL scale (i.e. the eddies below about $1000 \mathrm{~m}$ at the spatial scale and about $10 \mathrm{~min}$ at the temporal scale), the mean ergodic function $\operatorname{Ero}(A)$ and autocorrelation ergodic function $\operatorname{Er}(A)$ converge to 0 ; that is, they satisfy the conditions of mean and autocorrelation ergodic theorem. However, for the atmospheric turbulent eddies in excess of 2000-3000 $\mathrm{m}$ at the spatial scale and in excess of 30-60 min at the temporal scale, the mean ergodic function does not converge to 0 and, thus, dissatisfies the condition of mean ergodic theorem. Therefore, the turbulent eddies that are below the ABL scale belong to the wide ergodic stationary random processes, but the turbulent eddies that are larger than ABL scale belong to the non-ergodic random processes, or even the non-stationary random processes.

3. Due to above facts, when the stationary random process information of eddies below $10 \mathrm{~min}$ at the temporal scale and below $1000 \mathrm{~m}$ of ABL height at the spatial scale can be captured, the atmospheric turbulence may satisfy the condition of mean ergodic theorem. Therefore, an average of finite time can be used to substitute for the ensemble average to calculate the mean of random variable as measuring atmospheric turbulence with the eddy-covariance technique. But for the turbulence of eddies to be larger than $30 \mathrm{~min}$ at temporal scale (i.e. $2000 \mathrm{~m}$ at spatial scale magnitude), it dissatisfies the condition of mean ergodic theorem so that the eddycovariance technique cannot completely capture the information of non-stationary random processes. This will inevitably cause a high level of error when the average of finite time is used to substitute for the ensemble average in the experiments due to the loss of low-frequency component information associated with the large-scale eddies.

4. Although the atmospheric temperature stratification has different effects on the stability of eddies in the different scales, the ergodicity is mainly related to the eddy local stability, and its relation to the stratification stability of $\mathrm{ABL}$ is secondary.

5. The data series composed from seven stations compare with the observational data from a single station. The results show that the temporal and spatial scales of eddies belonging to the wide ergodic stationary random processes are extended from $10 \mathrm{~min}$ to below $30 \mathrm{~min}$ and from $1000 \mathrm{~m}$ to below $2000 \mathrm{~m}$, respectively. This signifies that the ergodic assumption is more likely to be satisfied well with multi-station observations, and observational results produced by the eddy-covariance technique are much closer to the true values when calculating the turbulence averages, variances or fluxes.

6. If the ergodic conditions of stationary random processes are more effectively satisfied, then the turbulence variances of eddies in the different temporal scale can comply with MOST very well; however, the turbulence variances of the non-ergodic random processes deviate from MOS relations.

Acknowledgements. This study is supported by project grant nos. 91025011 and 91437103 of the National Natural Science Foundation of China and project grant no. 2010CB951701-2 of the National Program on Key Basic Research. This work was strongly supported by Heihe Upstream Watershed Ecology-Hydrology Experimental Research Station, Chinese Academy of Sciences. We would like to express our sincere gratitude for their support. We also thank Gordon Maclean at NCAR for providing the detailed data of CASES-99 used in this study as well as the referees and editor very much for their heartfelt comments, discussions and marked errors.

Edited by: R. Sander 


\section{References}

Aubinet, M., Vesala, T., and Papale, D.: Eddy covariance, a practical guide to measurement and data analysis, Springer, Dordrecht, Heidelberg, London, New York, 438 pp., 2012.

Birkhoff, G. D.: Proof of the ergodic theorem, Proc. Nat. Acad. Sci. USA. 18, 656-660, 1931.

Boltzmann, L.: Analytischer beweis des zweiten Haubtsatzes der mechanischen Wärmetheorie aus den Sätzen über das Gleichgewicht der lebendigen Kraft, Wiener Berichte, 63, in: WAI, paper 20, 712-732, 1871.

Chang, S. S. and Huynh, G. D.: Analysis of sonic anemometer data from the CASES-99 field experiment, Army Research Laboratory, Adelphi, MD, 2002.

Chen, J., Hu Y., and Zhang L.: Principle of cross coupling between vertical heat turbulent transport and vertical velocity and determination of cross coupling coefficient, Adv. Atoms. Sci., 23, 639-648, 2007.

Chen, J., Hu, Y., Lu, S., and Yu, Ye.: Experimental demonstration of the coupling effect of vertical velocity on latent heat flux, Sci. China. Ser. D-Earth Sci., 56, 1-9, 2013.

Ehrenfest, P. and Ehrenfest-Afanassjewa, T.: The Conceptual Foundations of the Statistical Approach in Mechanics, New York, Cornell University Press, 1959.

Eichinger, W. E., Parlange, M. B., and Katul, G. G.: Lidar measurements of the dimensionless humidity gradient in the unstable ASL, Lakshmi, edited by: V., Albertson, J. and Schaake, J., Koster, R. D., and Duan, Q., Land Surface Hydrology, Meteorology, and Climate, American Geophysical Union, Washington, DC 7-13, 2001.

Foken, T. and Wichura, B.: Tools for quality assessment of surfacebased flux measurements, Agric Meteorol., 78, 83-105, 1996.

Foken, T., Göckede, M., Mauder, M., Mahrt, L., Amiro, B. D., and Munger, J. W.: Post-field data quality control, in: Handbook of micrometeorology: a guide for surface flux measurement and analysis, edited by: Lee, X., Massman, W. J., and Law, B.: Kluwer, Dordrecht, 181-208, 2004.

Foken, T., Aubinet, M., Finnigan, J. J., Leclerc, M. Y., Mauder, M., and Paw, U. K. T.: Results of a panel discussion about the energy balance closure correction for trace gases, Bull Am. Meteorol. Soc., 92, ES13-ES18, 2011.

Galanti, B. and Tsinober, A.: Is turbulence ergodic? Phys. Lett. A, 330, 173-18, 2004.

Higgins, C. W., Katul, G. G., Froidevaux, M., Simeonov, V., and Parlange, M. B.: Atmospheric surface layer flows ergodic?, Geophy. Res. Let., 40, 3342-3346, 2013.

Hill, R. J.: Implications of Monin-Obukhov similarity theory for scalar quantities, J. Atmos. Sci. 46, 2236-2244, 1989.

$\mathrm{Hu}$, Y.: Convergence movement influence on the turbulent transportation in atmospheric boundary layer, Adv. Atoms. Sci., 20, 794-798, 2003.

$\mathrm{Hu}$, Y., Chen, J., and Zuo, H.: Theorem of turbulent intensity and macroscopic mechanism of the turbulence development, Sci. China Ser. D-Earth Sci., 37, 789-800, 2007.

$\mathrm{Hu}$, Y. and Chen, J.: Nonequilibrium Thermodynamic Theory of Atmospheric Turbulence, in: Atmospheric Turbulence, Meteorological Modeling and Aerodynamics, edited by: Lang, P. R. and Lombargo, F. S., Nova Science Publishers, New York, 59-110, 2009.
Kaimal, J. C. and Wyngaard, J. C.: The Kansas and Minnesota experiments, Bound. Lay. Meteor., 50, 31-47, 1990.

Kaimal, J. C. and Gaynor, J. E.: Another look at sonic thermometry, Bound. Lay. Meteor., 56, 401-410, 1991.

Katul, G. G. and Hsieh, C. I.: A note on the flux-variance similarity relationships for heat and water vapor in the unstable atmospheric surface layer, Bound. Lay. Meteor., 90, 327-338, 1999.

Katul, G., Cava, D., Poggi, D., Albertson, J., and Mahrt, L.: Stationarity, homogeneity, and ergodicity in canopy turbulence, in: Handbook of micrometeorology: a guide for surface flux measurement and analysis, Lee, X., Massman, W., and Law, B., Kluwer Academic Publishers, Dordrecht/BOSTON/LONDON, 161-180, 2004.

Krengel, U.: Ergodic theorems, de Gruyter, Berlin, New York, 36 pp., 1985.

Lennaert van, V., Shigeo, K., and Genta, K.: Periodic motion representing isotropic turbulence, Fluid Dyn. Res., 38, 19-46, 2006.

Li, X., Hu, F., Pu, Y., Al-Jiboori, M. H., Hu, Z., and Hong, Z.: Identification of coherent structures of turbulence at the atmospheric surface layer, Adv. Atoms. Sci., 19, 687-698, 2002.

Martano, P.: Estimation of surface roughness length and displacement height from single-level sonic anemometer data, J. Appl. Meteorol., 39, 708-715, 2000.

Mattingly, J. C.: On recent progress for the stochastic Navier Stokes equations, Journées équations aux dérivées partielles, Univ. Nantes, Nantes, Exp. No. XI, 1-52, 2003.

Mauder, M., Desjardins, R. L., and MacPherson, J.: Scale analysis of airborne flux measurements over heterogeneous terrain in a boreal ecosystem, J. Geophys. Res., 112, D13112, doi:10.1029/2006JD008133, 2007.

McMillen, R. T.: An eddy correlation technique with extended applicability to non simple terrain, Bound. Lay. Meteor., 43, 231245, 1988.

Moore, C. J.: Frequency response corrections for eddy correlation systems, Bound. Lay. Meteor., 37, 17-35, 1986.

Neumann, J. V.: Proof of the quasi-ergodic hypothesis, Mathematics Proc. N. A. S., 18, 70-82, 1932.

Oncley, S. P., Friehe, C. A., Larue, J. C., Businger, J. A., Itsweire, E. C., and Chang, S. S.: Surface-Layer Fluxes, Profiles, and Turbulence Measurements over Uniform Terrain under Near-Neutral Conditions, J. Atmos. Sci., 53, 1029-1044, 1996.

Padro, J.: An investigation of flux-variance methods and universal functions applied to three land-use types in unstable conditions, Bound. Lay. Meteor., 66, 413-425, 1993.

Panofsky, H., A., Lenschow, D, H., and Wyngaard, J. C.: The characteristics of turbulent velocity components in the surface layer under unstable conditions, Bound. Lay. Meteor., 11, 355-361, 1977.

Papoulis, A. and Pillai, S. U.: Probability, random variables and stochastic processes, McGraw-Hill, New York, 666 pp., 1991.

Poulos, G. S., Blumen, W., Fritts, D, C., Lundquist, J. K., Sun, J., Burns, S. P., Nappo, C., Banta, R., Newsom, R., Cuxart, J., Terradellas, E., and Balsley, Ben.: CASES-99: a comprehensive investigation of the stable nocturnal boundary layer, Bull. Amer. Meteor. Soc., 83, 555-581, 2002.

Schotanus, P., Nieuwstadt, F. T. M., and de Bruin, H. A. R.: Temperature measurement with a sonic anemometer and its application to heat and moisture fluxes, Bound. Lay. Meteor., 26, 81-93, 1983. 
Stull, R. B.: An introduction to boundary layer meteorology, Kluwer Academic Publ, Dordrecht, 670 pp., 1988.

Uffink, J.: Boltzmann's work in statistical physics, Stanford encyclopedia of philosophy, Edward, N. Z., 2004.

Vickers, D. and Mahrt, L.: Quality control and flux sampling problems for tower and aircraft data, J. Atmos. Oceanic. Technol., 14, 512-526, 1997.

Webb, E. K., Pearman, G. I., and Leuning, R.: Correction of the flux measurements for density effects due to heat and water vapor transfer, Q. J. R. Meteorol. Soc., 106, 85-100, 1980.
Wilczak, J. M., Oncley, S. P., and Stage, S. A.: Sonic anemometer tilts correction algorithms. Bound. Lay. Meteor., 99, 127-150, 2001.

Wyngaard, J. C.: Turbulence in the atmosphere, getting to know turbulence, Cambridge University Press, New York, 393 pp., 2010.

Zuo, H., Xiao X., Yang Q., Dong L., Chen J., and Wang S.: On the atmospheric movement and the imbalance of observed and calculated energy in the surface layer, Sci. China. Ser. D-Earth Sci., 55, 1518-1532, 2012. 